\title{
INVESTIGATION OF DAMPING COEFFICIENT FOR MAGNETORHEOLOGICAL ELASTOMER
}

\author{
M.N.Hazwan Hadzir ${ }^{1}$, Z.A.Norfaidayu' ${ }^{1}$, M.S.Mohamad Sabri ${ }^{1}$, Muhamad-Husaini \\ Abu-Bakar ${ }^{*}$ \\ ${ }^{1}$ System Engineering and Energy Laboratory, \\ Universiti Kuala Lumpur, \\ Malaysian Spanish Institute, \\ Kulim Hi-tech Park,09000, \\ Kulim,Kedah, Malaysia. \\ *Email: muhamadhusaini@ unikl.edu.my \\ Phone: +6044035199; Fax: +6044035201
}

\begin{abstract}
Magnetorheological elastomer (MRE) is a smart material where its stiffness vary with presence of magnetic field.These special properties lead to various application as mechanical devise such as damper. Damping coefficient is criteria that need to consider while designing the device. This paper aims to study the effect of iron filling and elastomer composition on a damping coefficient of MRE. Five MRE specimens with different CIP composition from 30 to $70 \%$ was prepared.the speciment was tested with testbed which is has a Constance distance permanent magnet $(5 \mathrm{~mm})$ and motor speed $(131.9 \mathrm{~Hz})$. The vibration amplitude in frequency domain was used to calculate the damping coefficient by half-power bandwidth equation. Experimental result indicates that the highest damping coefficient is at 30\% CIP concentration and the lowest is at $70 \%$ CIP composition. As a conclusion, the damping coeficiet is decrease with increasing in CIP composition.this finding useful to design an optimal active damper for mechanical component.
\end{abstract}

Keywords: Magnetorheological elastomer, smart material, damping coefficient, semiactive damper, damping properties.

\section{INTRODUCTION}

MRE was categorized as an intelligent material due to ability in reclaims its shape when a magnetic field is removed. MRE physical state can be tuned between a soft elastomer and a semi-solid with varying an external magnetic field induced to the material(Chen \& Liao, 2007). MRE has overcome some the problem on MRF, such as deposition, sealing issue and environmental contamination. The pioneer work on the magnetically sensitive elastomer was firstly reported by (Rigbi \& Jilkén, 1983).

MRE consist of three necessary components: magnetic particle (carbonyl iron), an elastomer, and additives (usually silicon oil.). The magnetic particle size such as carbonyl iron commonly is lie in between 5-10 micron. The Elastomer such as silicon rubber used as they are soft or deformable at room temperature, the elastomer can have the ability to extend from 5-700\%, depending on the specific material. (Ruddy, Ahearne, \& Byrne, 2012). The component is mixed together to form a compound with 
significant density magnetic particle randomly dispersed or pre-arrange in a low-density matrix. In the absence of magnetic field, the magnetic particle will attract each other as shown in figure 1.

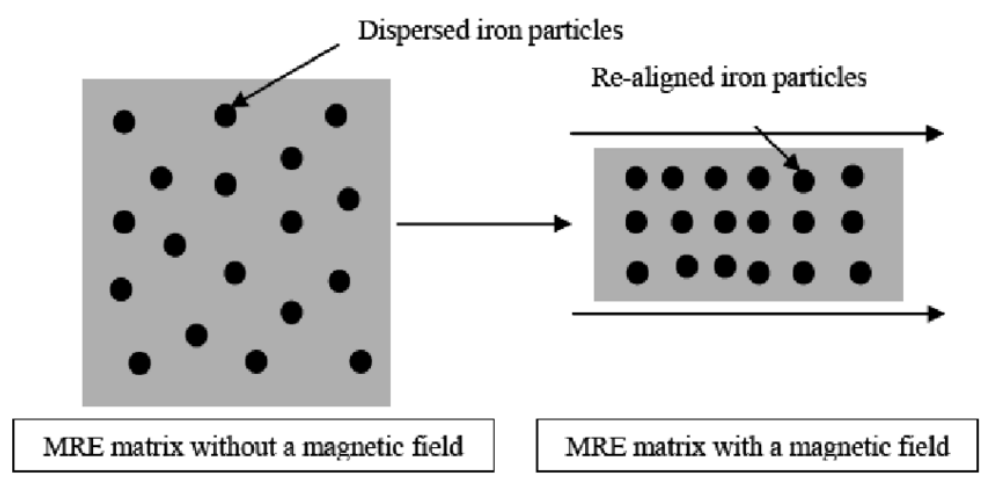

Figure 1. Schematic diagram of MRE (Usman \& Jung, 2015)

Magneto-rheological elastomer(MRE) has their specialties compare with MR fluid and conventional device nowadays (Wereley, 2013). MRE can work in multiple degrees of freedom (DOF) while MR fluid work in a single degree of freedom. Magnetorheological elastomer device work in the pre-yield region, while MR fluid work in the post-yield region. Thus, MRE usually can work with variable stiffness while MR Fluid device works in variable damping. Due to able work in variable stiffness when the magnetic field applied, the most researcher looking potential in MRE to develop smart devices such as vibration isolation, a vibration absorber, magnetic sensor and actuator (Y. Li, Li, Li, \& Du, 2014).

The mechanical and rheological properties of an elastomeric material containing magnetizable particles can be changed reversibly by applying an external magnetic field. This is called the magnetorheological effect. The origin of the field dependence of the MRE properties is the existence of field-induced dipole magnetic forces between the particles. Therefore, the mechanical behavior of MR elastomer is a combination of matrix properties and the properties of magnetizable particle formations inside the matrix.

Dynamic mechanical properties testing has been used for characterizing the shear storage modulus of MR elastomer (Ismail, Ibrahim, Hamid, Mahmood, \& Adnan, 2016). Most of the test have been done by the use of force oscillation (Huang, Yan, Liu, $\mathrm{Li}$, \& Wang, 2015). Recently, free vibration techniques have also been used in MRE studies (G. H. Li, Huang, Gu, \& Wang, 2013). Perhaps, the researcher investigated the modulus storage of the MRE and shear stress of the material to characterize the properties of the material. G. H. Li et al., (2013) was propose the new method of preparation MRE specimen and new approach to identify the mechanical properties of MRE. Shuib \& Pickering, (2016) use Dynamic mechanical properties testing to identify dynamic properties of MRE based on iron sand and natural rubber. Kukla, Górecki, Malujda, Talaśka, \& Tarkowski, (2017) use compression method to identify the behavior and properties of MRE. MRE was considered as a smart material because it is able to control the stiffness by controlling the magnetic field. Thus, the investigation of damping coefficient for MRE is needed for optimizing the application in the featured. 
This paper, isotropic MRE specimen was prepared by various percentage of Carbonyl Iron Particle (CIP) composition from 30\% to 70\%. The accelerometer sensor was used to measure the vibration in every CIP composition. The damping coefficient was calculated by half-power bandwidth equation.

\section{METHODOLOGY}

\section{Magnetorheological Elastomer (MRE) Preparation}

In this section, the preparation of Magnetorheological Elastomer specimens for experimental are explained. The specimen was prepared by following the portion in table 1; there are four material components were used in the preparation of MRE specimens, which is Carbonyl iron particle (CIP), Natural silicon rubber, grease and silicone oil. The portion of composition was allocated following the volume of MRE mold in this experiment.

Table 1. The composition of MRE specimens

$\begin{array}{ccccc}\text { \% of CIP } & \text { Rubber(gram) } & \text { Grease(gram) } & \text { Silicone Oil } & \text { CIP(gram) } \\ & & & \text { (gram) } & \\ \mathbf{3 0} & 84.9 & 6.06 & 12.13 & 36.4 \\ \mathbf{4 0} & 88.5 & 7.38 & 14.76 & 59.02 \\ \mathbf{5 0} & 86.92 & 8.69 & 17.32 & 86.92 \\ \mathbf{6 0} & 80.04 & 10.00 & 20.01 & 120.06 \\ \mathbf{7 0} & 67.91 & 11.32 & 22.63 & 158.46\end{array}$

The MRE specimen fabrication procedure starts with mixing the grease and the natural silicone rubber in a bowl during 10min to obtain an elastomer gel with good homogenization. Next, pour silicon oil and continues mixing in 10 minutes until the mixer homogenize. Lastly, pour mixing for 15 minutes until it is homogenization. The mixer MRE is loaded into the mold and stored for 24 hours at room temperature. 


\section{Experimental Setup}

Figure 2 shows the experimental setup for investigation of MRE damping coefficient. Figure 2(a) shows the controller component for controlling the motor speed including the Arduino, MD10C motor driver and battery as an energy source for Arduino. Figure 2(b) is the MRE damping coefficient setup; this setup has three component, DC motor as vibration source, MRE specimens, and a permanent magnet. The piezoelectric accelerometer sensor was used to measure the vibration. The signal from accelerometer sensors are recorded using LMS SCADAS hardware, and then the data is analyzed using LMS Test.Lab software to generate the vibration figures and graphs data. The experiment was conducted by several of MRE specimens from $30 \%$ to $70 \%$ CIP filling.

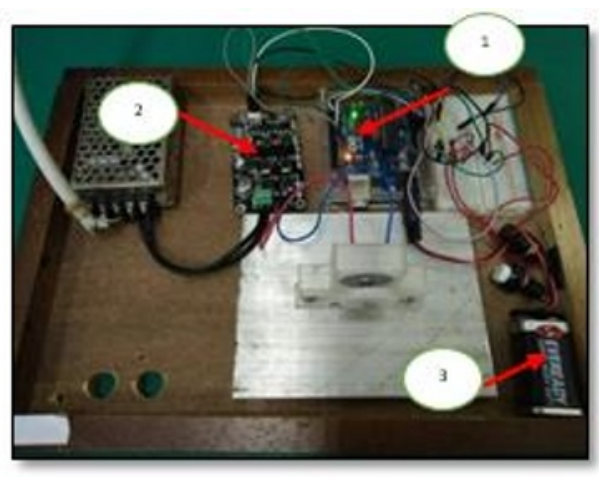

(a)

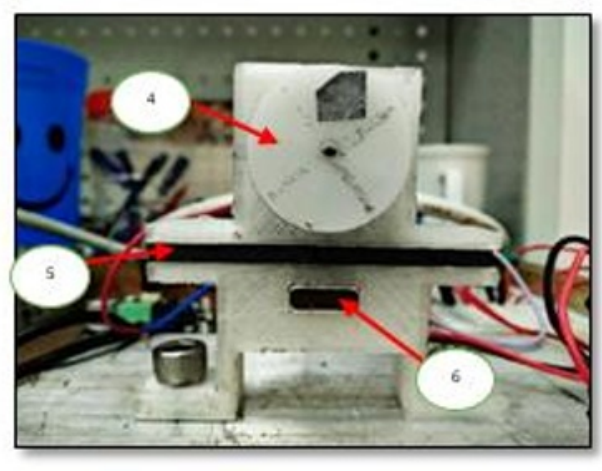

(b)

Figure 2. (a) Controller setup(1)Arduino,(2)MD10C Motor Driver,(3)Battery; (b) vibration setup (4)DC motor,(5)MRE specimen,(6)Permanent Magnet

\section{Half-power bandwidth equation.}

The equation to calculate the damping coefficient is by using the half-power bandwidth method in equation 1 .

$$
\frac{\omega_{2}-\omega_{1}}{\omega_{n}}=2 \zeta
$$

Where $\omega$ (omega) is angular frequency, and $\zeta($ zeta) is damping coefficient. 


\section{RESULTS AND DISCUSSION}

\section{Frequency Domain Analyses}

Figure 3 shows the vibration amplitude comparison by various CIP composition. The data was collected by LMS SCADAS hardware and piezoelectric accelerometer sensor. The data was analyzed by LMS Test.lab to generate frequency domain graph for further investigation. The graph in figure 3 shows, all the components are difference amplitude for every specimen. The higher amplitude is at 70\% CIP composition with pick value $0.116 \mathrm{~g}$. The lowers amplitude in this graph is at $30 \% \mathrm{CIP}$ composition with pick value $0.0147 \mathrm{~g}$. The amplitude keeps increasing proportional increasing the CIP composition due to the increase of stiffness. However, the non-linearity was detected in $60 \%$ CIP composition; the amplitude drastically drop lower than 50\% CIP composition and $40 \%$ CIP composition event the test was repeated in 5 times, his phenomena is the new contribution in MRE for further study.

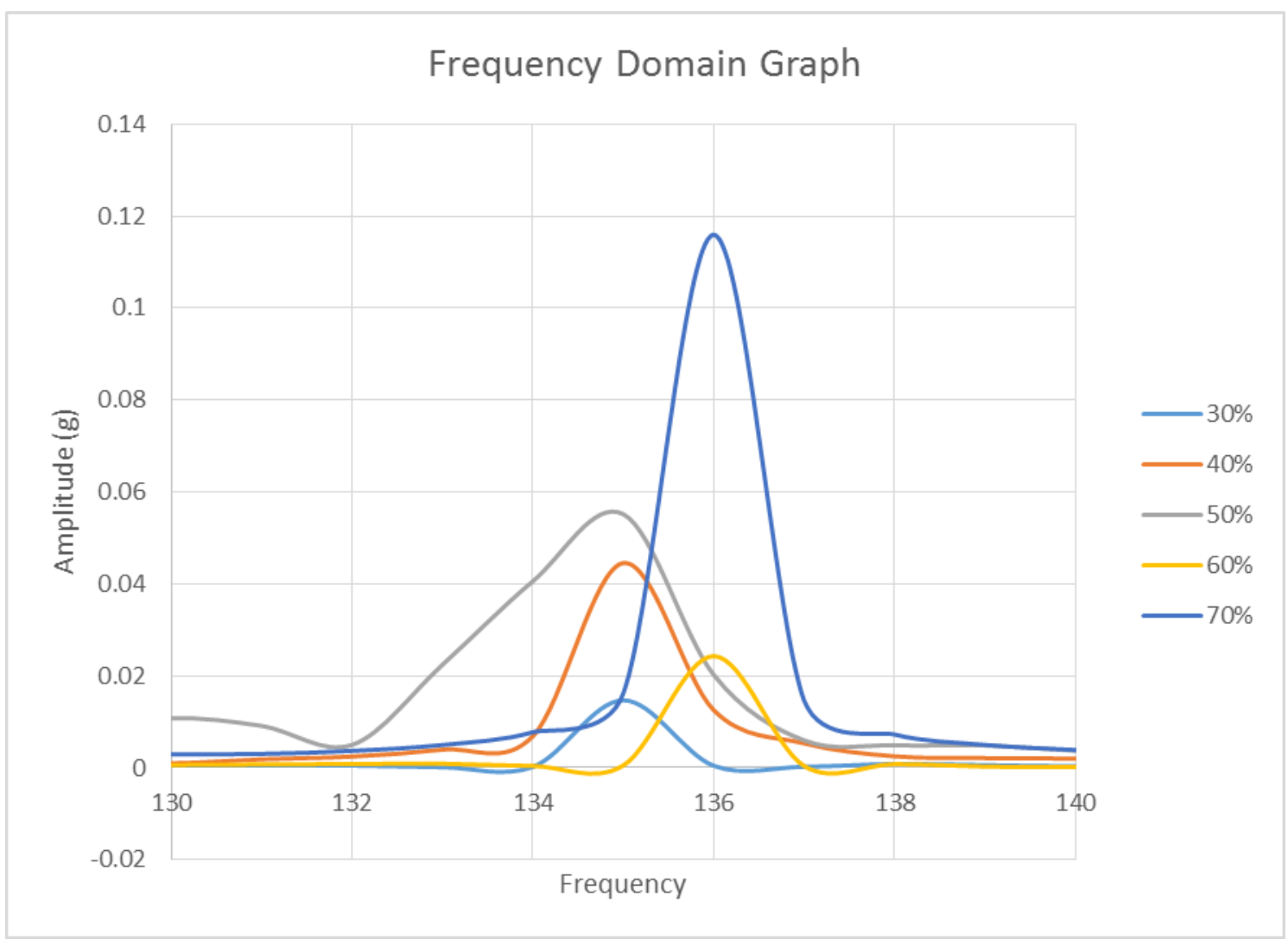

Figure 3. Vibration comparison

Figure 4 shows the damping coefficient value for a different CIP composition in MRE. The damping coefficient value calculates as explained in equation 1 . The graph in figure 4 shows that maximum value of damping coefficient occurs at CIP with $30 \%$ and the value was 0.1988 . There was no significant difference in damping coefficient value when the CIP percentage varied from $40 \%$ to $60 \%$. However, the damping coefficient value dropped drastically when the CIP in MRE was 70\%.the span of damping coefficient was 0.0728 which is maximum 0.1988 and minimum 0.126 . The factor that contributes to the high value of damping coefficient for CIP $30 \%$ is it stiffness are lowest as compare to other CIP composition. When the CIP \% is high, the magnetic 
field permeability is increased, and the internal force in the MRE increase. The force is increased because the distance between the CIP is closer and create a better magnetic flux flow path.

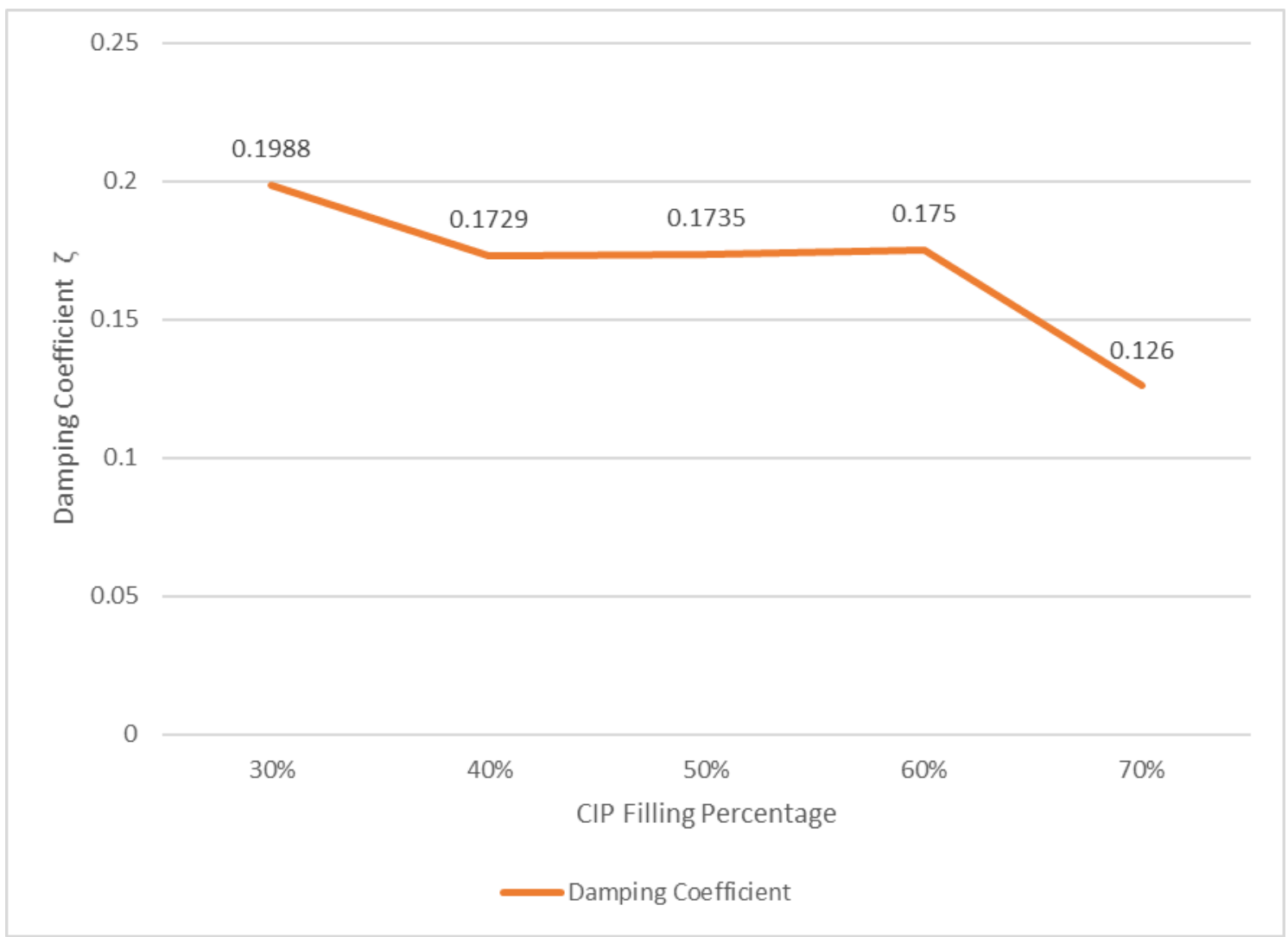

Figure 4. Damping coefficient

\section{CONCLUSION}

Experiment result reveals the effect of CIP on damping coefficient of MRE. MRE with $30 \%$ CIP shows the highest damping coefficient value compare to other composition. The stiffness was decreased with increases in CIP composition in MRE. As a conclusion; the MRE damping coefficient was inversely proportional to CIP composition. This finding useful to design a mechanical device such as damper and vibration isolator. Because the by magnetic field influence the MRE damping coefficient, the experiment should extend to the different level of magnetic field magnitude.

\section{ACKNOWLEDGEMENTS}

The authors would like to acknowledge Universiti Kuala Lumpur, Malaysian Spanish Institute, System Engineering and Energy Laboratory UniKL Msi and Dynamic Vibration Laboratory UniKL Msi for providing the consultation, technical support, and give permission to use the equipment facilities in their Laboratory for make sure this project is successful. 


\section{REFERENCES}

[1] Chen, J., \& Liao, W. H. (2007). Design and control of a magnetorheological actuator for leg exoskeleton. 2007 IEEE International Conference on Robotics and Biomimetics, ROBIO, 1388-1393. https://doi.org/10.1109/ROBIO.2007.4522367

[2] Huang, X. G., Yan, Z. Y., Liu, C., Li, G. H., \& Wang, J. (2015). Study on the resistance properties of magnetorheological elastomer. Materials Research Innovations, 19(sup5), S5-924-S5-928. https://doi.org/10.1179/1432891714Z.0000000001223

[3] Ismail, R., Ibrahim, A., Hamid, H. A., Mahmood, M. R., \& Adnan, A. (2016). Dynamic mechanical behavior magnetorheological nanocomposites containing CNTs: A review. AIP Conference Proceedings, 1733. https://doi.org/10.1063/1.4948878

[4] Kukla, M., Górecki, J., Malujda, I., Talaśka, K., \& Tarkowski, P. (2017). The Determination of Mechanical Properties of Magnetorheological Elastomers (MREs). Procedia Engineering, 177, 324-330. https://doi.org/10.1016/j.proeng.2017.02.233

[5] Li, G. H., Huang, X. G., Gu, X. Y., \& Wang, J. (2013). Fabrication and Mechanical Properties Study of the Magnetorheological Elastomer. Applied Mechanics and Materials, 376, 148-152. https://doi.org/10.4028/www.scientific.net/AMM.376.148

[6] Li, Y., Li, J., Li, W., \& Du, H. (2014). A state-of-the-art review on magnetorheological elastomer devices. Smart Materials and Structures, 23(12), 123001. https://doi.org/10.1088/0964-1726/23/12/123001

[7] Rigbi, Z., \& Jilkén, L. (1983). The response of an elastomer filled with soft ferrite to mechanical and magnetic influences. Journal of Magnetism and Magnetic Materials, 37(3), 267-276. https://doi.org/10.1016/0304-8853(83)90055-0

[8] Ruddy, C., Ahearne, E., \& Byrne, G. (2012). A review of Magnetorheological Elastomers: Properties and Applications. Advanced Manufacturing Science (AMS) Research, 20, 1-7.

[9] Shuib, R. K., \& Pickering, K. L. (2016). Effect of Carbon Black on the Dynamic Properties of Anisotropic Magnetorheological Elastomer, 12, 1-12.

[10] Usman, M., \& Jung, H.-J. (2015). Recent developments of magneto-rheological elastomers for civil engineering applications.

[11]Wereley, N. M. (2013). Magnetorheology. https://doi.org/10.1039/9781849737548 